\title{
On a quadratic transformation due to Exton and its generalization
}

\author{
Gradimir V. Milovanović¹ (D), Arjun K. Rathie ${ }^{2}$ (I) \\ ${ }^{1}$ Serbian Academy of Sciences and Arts, 11000 Beograd, Serbia \& University of Niš, Faculty of Sciences \\ and Mathematics, 18000 Niš, Serbia \\ ${ }^{2}$ Department of Mathematics, Vedant College of Engineering and Technology (Rajasthan Technical \\ University), Bundi, Rajasthan State, India
}

\begin{abstract}
In 2003, Exton established numerous quadratic transformation formulas. The aim of this short note is to provide generalization of one of the quadratic transformation formulas.
\end{abstract}

Mathematics Subject Classification (2010). 33C20

Keywords. Quadratic transformation, Bailey's transform, Kummer's summation theorem, generalizations

\section{Introduction}

The generalized hypergeometric function ${ }_{p} F_{q}(a ; b ; z)$, where $a=\left(a_{1}, a_{2}, \ldots, a_{p}\right)$ and $b=\left(b_{1}, b_{2}, \ldots, b_{p}\right)$, has series expansion $[13$, p. 41$]$

$$
{ }_{p} F_{q}\left[\begin{array}{c}
a_{1}, a_{2}, \ldots, a_{p} \\
b_{1}, b_{2}, \ldots, b_{p}
\end{array} ; z\right]=\sum_{n=0}^{\infty} \frac{\left(a_{1}\right)_{n}\left(a_{2}\right)_{n} \ldots\left(a_{p}\right)_{n}}{\left(b_{1}\right)_{n}\left(b_{2}\right)_{n} \ldots\left(b_{q}\right)_{n}} \frac{z^{n}}{n !},
$$

where for nonnegative $n \in \mathbb{N}_{0}$, the Pochhammer symbol is defined by $(a)_{0}=1,(a)_{n}=$ $a(a+1) \cdots(a+n-1)$.

The generalized hypergeometric functions ${ }_{p} F_{q}$, and in particular hypergeometric functions ${ }_{2} F_{1}$, are fundamental in the field of applied mathematics, mathematical physics and engineering mathematics. Most of the commonly used functions that occur in analysis are special cases or limiting cases of hypergeometric functions.

In Wolfram's MATHEMATica this important function ${ }_{p} F_{q}$ is implemented as HypergeometricPFQ and suitable for both symbolic and numerical calculation. For $p=q+1$, it has a branch cut discontinuity in the complex $z$ plane running from 1 to $\infty$. When $p \leq q$ the above series on the right-hand side converges for each $z \in \mathbb{C}$.

It should be remarked here that whenever hypergeometric function or generalized hypergeometric function can be summed to be expressed in terms of gamma function, the results are very important from the application point of view. Thus the classical summation theorems such as those of Gauss, Gauss second, Kummer and Bailey for the series ${ }_{2} F_{1}$; Watson, Dixon, Whipple and Saalschütz for the series ${ }_{3} F_{2}$ and others play a key role.

\footnotetext{
*Corresponding Author.

Email addresses: gvm@mi.sanu.ac.rs (G.V. Milovanović), arjunkumarrathie@gmail.com (A.K. Rathie)

Received: 24.02.2018; Accepted: 24.06.2018
} 
A recent study of generalized summation theorems for the series ${ }_{2} F_{1}$ with an applications to Laplace transforms of convolution type integrals involving Kummer's functions ${ }_{1} F_{1}$ has been given [10].

Bailey [2], in his very interesting, useful and popular paper, obtained a large number of results involving the products of the generalized hypergeometric function. Also, interesting summations due to Ramanujan can also be obtained very quickly with help of above mentioned classical summation theorems.

In addition to this, in 1982, Gessel and Stanton [7] have evaluated some interesting and strange evaluation of the hypergeometric function.

Recently good deal of progress had been done in the direction of generalizing the above mentioned classical summation theorems. For this, we refer a paper by Rakha and Rathie [12].

Very recently, by means of telescoping method, Chu [4] presented a new proof for a ${ }_{2} F_{1}$-series identity conjectured by Gosper [8] and was proved in 2013 by Ebisu [5].

On the other hand, as a consequence of Bailey's transform, Slater [13, Eq. (2.4.10), p. 60] gave a useful and general hypergeometric transformation which was recently corrected by Kim, et. al [9] and is given by

$$
\begin{aligned}
\sum_{n=0}^{\infty} & \frac{((a))_{n}((d))_{n}((v))_{2 n}}{((h))_{n}((g))_{n}((f))_{2 n}} \frac{x^{n} y^{n} z^{2 n}}{n !} U+D+V F_{E+F+G}\left[\begin{array}{l}
(u),(d)+n,(v)+2 n \\
(e),(g)+n,(f)+2 n
\end{array} ; x w z\right] \\
= & \sum_{n=0}^{\infty} \frac{((d))_{n}((u))_{n}((v))_{n}}{((e))_{n}((f))_{n}((g))_{n}} \frac{x^{n} w^{n} z^{n}}{n !} \\
& \times_{A+E+V+1} F_{U+H+F}\left[\begin{array}{c}
-n,(a), 1-n-(e),(v)+n \\
(h), 1-n-(u),(f)+n
\end{array} ;(-1)^{1+E-U} \frac{y z}{w}\right],
\end{aligned}
$$

where we have adopted the convention of writing the finite sequence of parameters $\left(a_{1}, \ldots, a_{A}\right)$ simply by $(a)$ and the product of Pochhammer symbols by $((a))_{n}=$ $\left(a_{1}\right)_{n} \cdots\left(a_{A}\right)_{n}$, where an empty product is understood to be unity. The previous general result contains as special cases many relationships between generalized hypergeometric functions.

In our present investigations, we are interested in the following special case

$$
\begin{aligned}
\sum_{n=0}^{\infty} \frac{((a))_{n}((d))_{n}((v))_{2 n}}{((h))_{n}((g))_{n}((f))_{2 n}} \frac{x^{n} y^{n}}{n !} & \\
= & \sum_{n=0}^{\infty} \frac{((d))_{n}((u))_{n}((v))_{n}}{((e))_{n}((f))_{n}((g))_{n}} \frac{x^{n}}{n !} F_{E+F+G}\left[\begin{array}{c}
(u),(d)+n,(v)+2 n \\
(e),(g)+n,(f)+2 n
\end{array} ; x\right] \\
& \times_{A+E+V+1} F_{U+H+F}\left[\begin{array}{c}
(a), 1-n-(e),(v)+n,-n \\
(h), 1-n-(u),(f)+n
\end{array} ;(-1)^{1+E-U} y\right],
\end{aligned}
$$

Further, if we take $D=2, F=1, U=V=E=G=0$ with $d_{1}=d-1 / 2, d_{2}=d$ and $f=2 d$, we obtained the following corrected version of general quadratic transformation given by Exton [6, Eqn. (2.1)],

$$
\begin{aligned}
& \sum_{n=0}^{\infty} \frac{((a))_{n}\left(d-\frac{1}{2}\right)_{n}}{((h))\left(d+\frac{1}{2}\right)_{n} 4^{n}} \frac{x^{n} y^{n}}{n !}{ }_{2} F_{1}\left[\begin{array}{c}
d+n-\frac{1}{2}, d+n \\
2(d+n)
\end{array} ; x\right] \\
& =\sum_{n=0}^{\infty} \frac{\left(d-\frac{1}{2}\right)_{n}(d)_{n}}{(2 d)_{n}} \frac{x^{n}}{n !}{ }^{A+1} F_{H+1}\left[\begin{array}{c}
(a),-n \\
(h), 2 d+n
\end{array} ;-y\right]
\end{aligned}
$$

because

$$
\frac{((d))_{n}}{((f))_{2 n}}=\frac{\left(d-\frac{1}{2}\right)_{n}(d)_{n}}{(2 d)_{2 n}}=\frac{\left(d-\frac{1}{2}\right)_{n}}{4^{n}\left(d+\frac{1}{2}\right)_{n}} .
$$


In this, if we use the result [14, p. 34]

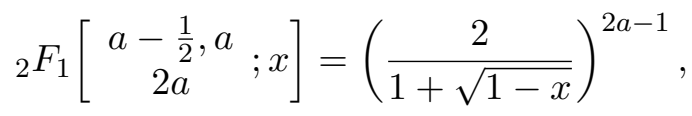

we get the following result also obtained by Exton [6],

$$
\begin{aligned}
& \left(\frac{2}{1+\sqrt{1-x}}\right)^{2 d-1}{ }_{A+1} F_{H+1}\left[\begin{array}{r}
(a), d-\frac{1}{2} \\
(h), d+\frac{1}{2}
\end{array} ; y z\right] \\
& \quad=\sum_{n=0}^{\infty} \frac{\left(d-\frac{1}{2}\right)_{n}(d)_{n}}{(2 d)_{n}} \frac{x^{n}}{n !} A_{+1} F_{H+1}\left[\begin{array}{c}
(a),-n \\
(h), 2 d+n
\end{array} ;-y\right],
\end{aligned}
$$

where

$$
z=\frac{x}{(1+\sqrt{1-x})^{2}} .
$$

Now from (1.2), we observe that if the inner hypergeometric function on the right of (1.2) can be summed by means of known summation theorems, then the resulting quadratic transformations are obtained. Thus, for example, if in (1.2), we take $A=1, H=0, y=1$ and $a=2 d-1$, we have

$$
\begin{aligned}
& \left(\frac{2}{1+\sqrt{1-x}}\right)^{2 d-1}{ }_{2} F_{1}\left[\begin{array}{c}
2 d-1, d-\frac{1}{2} \\
d+\frac{1}{2}
\end{array} ;\right] \\
& \quad=\sum_{n=0}^{\infty} \frac{\left(d-\frac{1}{2}\right)_{n}(d)_{n}}{(2 d)_{n}} \frac{x^{n}}{n !}{ }_{2} F_{1}\left[\begin{array}{c}
2 d-1,-n \\
2 d+n
\end{array} ;-1\right],
\end{aligned}
$$

where $z$ is given by (1.3).

The function ${ }_{2} F_{1}$ appearing on the right hand side of (1.4) can be evaluated with the help of classical Kummer's summation theorem [14] viz,

$$
{ }_{2} F_{1}\left[\begin{array}{c}
a, b \\
1+a-b
\end{array} ;-1\right]=\frac{\Gamma\left(1+\frac{a}{2}\right) \Gamma(1+a-b)}{\Gamma(1+a) \Gamma\left(1+\frac{a}{2}-b\right)},
$$

and after some simplification, we get the following result

$$
\left(\frac{2}{1+\sqrt{1-x}}\right)^{2 d-1}{ }_{2} F_{1}\left[\begin{array}{c}
2 d-1, d-\frac{1}{2} \\
d+\frac{1}{2}
\end{array} ; z\right]={ }_{2} F_{1}\left[\begin{array}{c}
d-\frac{1}{2}, d \\
d+\frac{1}{2}
\end{array} ; x\right],
$$

where $z$ is given by (1.3). This formula was also obtained by Choi and Rathie [3],

The aim of this short note is to provide generalization of the result (1.5) in the most general form. For this we will use the results known as the generalizations of Kummer's summation theorem obtained earlier by Rakha and Rathie [12].

\section{Generalizations of (1.5)}

In this section, we prove two master formulas for the quadratic transformation (1.5).

Proposition 2.1. For each $i \in \mathbb{N}_{0}$ the following formulas

$$
\begin{aligned}
\left(\frac{2}{1+\sqrt{1-x}}\right)^{2 d-1} & { }_{2} F_{1}\left[\begin{array}{c}
2 d+i-1, d-\frac{1}{2} \\
d+\frac{1}{2}
\end{array} z\right] \\
& =2^{-i} \sum_{r=0}^{i}\left(\begin{array}{l}
i \\
r
\end{array}\right){ }_{2} F_{1}\left[\begin{array}{c}
d-\frac{1}{2}, d+\frac{r}{2} \\
d+\frac{1}{2}
\end{array}\right]
\end{aligned}
$$


and

$$
\begin{aligned}
& \left(\frac{2}{1+\sqrt{1-x}}\right)^{2 d-1}{ }_{2} F_{1}\left[\begin{array}{c}
2 d-i-1, d-\frac{1}{2} \\
d+\frac{1}{2}
\end{array} z\right] \\
& \quad=\frac{(-2)^{i}}{\Gamma(i+1)} \sum_{r=0}^{i}(-1)^{r}\left(\begin{array}{l}
i \\
r
\end{array}\right) \frac{\Gamma\left(d+\frac{r}{2}\right)}{\Gamma\left(d-i+\frac{r}{2}\right)}{ }_{3} F_{2}\left[\begin{array}{c}
1, d-\frac{1}{2}, d+\frac{r}{2} \\
i+1, d+\frac{1}{2}
\end{array} ; x\right]
\end{aligned}
$$

hold, where

$$
z=\frac{x}{(1+\sqrt{1-x})^{2}} .
$$

Proof. The proofs of these formulas are quite straight forward. For this in order to prove (2.1), if we take $A=1, H=0, y=1$ and $a=2 d+i-1$ in (1.1), then for $z=x /(1+\sqrt{1-x})^{2}$ and $i=0,1,2, \ldots$, we have

$$
\begin{aligned}
& \left(\frac{2}{1+\sqrt{1-x}}\right)^{2 d-1}{ }_{2} F_{1}\left[\begin{array}{c}
2 d+i-1, d-\frac{1}{2} \\
d+\frac{1}{2}
\end{array} ;\right] \\
& \quad=\sum_{n=0}^{\infty} \frac{\left(d-\frac{1}{2}\right)_{n}(d)_{n}}{(2 d)_{n}} \frac{x^{n}}{n !}{ }_{2} F_{1}\left[\begin{array}{c}
2 d+i-1,-n \\
2 d+n
\end{array} ;-1\right] .
\end{aligned}
$$

We now observe that the ${ }_{2} F_{1}$ appearing on the right hand side of the last formula can be evaluated with the help of generalized Kummer's summation theorem [12],

$$
\begin{array}{r}
{ }_{2} F_{1}\left[\begin{array}{c}
a, b \\
1+a-b-i
\end{array} ;-1\right]=\frac{2^{-a} \Gamma\left(\frac{1}{2}\right) \Gamma(1+a-b-i)}{\Gamma\left(\frac{1}{2}(1+a-2 b-i)\right) \Gamma\left(\frac{1}{2}(2+a-2 b-i)\right)} \\
\times \sum_{r=0}^{i}\left(\begin{array}{c}
i \\
r
\end{array}\right) \frac{\Gamma\left(\frac{1}{2}(a-2 b-i+r+1)\right)}{\Gamma\left(\frac{1}{2}(a-i+r+1)\right)},
\end{array}
$$

taking $a=2 d+i-1$ and $b=-n$, i.e.,

$$
{ }_{2} F_{1}\left[\begin{array}{c}
2 d+i-1,-n \\
2 d+n
\end{array} ;-1\right]=\frac{2^{-(2 d+i-1)} \Gamma\left(\frac{1}{2}\right) \Gamma(2 d+n)}{\Gamma(d+n) \Gamma\left(\frac{1}{2}+d+n\right)} \sum_{r=0}^{n}\left(\begin{array}{c}
i \\
r
\end{array}\right) \frac{\Gamma\left(d+n+\frac{r}{2}\right)}{\Gamma\left(d+\frac{r}{2}\right)} .
$$

Using the functional equality $\Gamma(z+n)=(z)_{n} \Gamma(z)$ and applying Legendre's duplication formula for the gamma function (cf. [1, p. 256]),

$$
\Gamma(2 d)=\frac{2^{2 d-1}}{\sqrt{\pi}} \Gamma(d) \Gamma\left(d+\frac{1}{2}\right),
$$

we obtain

$$
{ }_{2} F_{1}\left[\begin{array}{c}
2 d+i-1,-n \\
2 d+n
\end{array} ;-1\right]=\frac{2^{-i}(2 d)_{n}}{(d)_{n}\left(d+\frac{1}{2}\right)_{n}} \sum_{r=0}^{n}\left(\begin{array}{l}
i \\
r
\end{array}\right)\left(d+\frac{r}{2}\right)_{n} .
$$

Now, the right hand side of (2.3) reduces to

$$
\sum_{n=0}^{\infty} \frac{\left(d-\frac{1}{2}\right)_{n}}{\left(d+\frac{1}{2}\right)_{n}} \frac{x^{n}}{n !} \frac{1}{2^{i}} \sum_{r=0}^{n}\left(\begin{array}{l}
i \\
r
\end{array}\right)\left(d+\frac{r}{2}\right)_{n}=\frac{1}{2^{i}} \sum_{r=0}^{i}\left(\begin{array}{l}
i \\
r
\end{array}\right){ }_{2} F_{1}\left[\begin{array}{c}
d-\frac{1}{2}, d+\frac{r}{2} ; x \\
d+\frac{1}{2}
\end{array}\right] .
$$

Thus, this completes the proof of (2.1). 
In a similar manner, the result (2.2) can be proved with the help of the summation formula

$$
\begin{array}{r}
{ }_{2} F_{1}\left[\begin{array}{c}
a, b \\
1+a-b+i
\end{array} ;-1\right]=\frac{2^{-a} \Gamma\left(\frac{1}{2}\right) \Gamma(1+a-b+i) \Gamma(b-i)}{\Gamma(b) \Gamma\left(\frac{1}{2}(1+a-2 b+i)\right) \Gamma\left(\frac{1}{2}(2+a-2 b+i)\right)} \\
\times \sum_{r=0}^{i}(-1)^{r}\left(\begin{array}{c}
i \\
r
\end{array}\right) \frac{\Gamma\left(\frac{1}{2}(a-2 b+i+r+1)\right)}{\Gamma\left(\frac{1}{2}(a-i+r+1)\right)},
\end{array}
$$

obtained recently also by Rakha and Rathie [12] for each $i \in \mathbb{N}_{0}$.

\section{Special cases}

We mention here a few special cases of formulas given in Proposition 2.1.

For particular values of $i$ we have:

(1) Taking $i=0$, the both of formulas, (2.1) and (2.2), reduce to (1.5) due to Exton [6].

(2) For $i=1,2,3,4,5$ formulas (2.1) and (2.2) give results obtained earlier by Pogany and Rathie [11].

Similarly other results can be obtained.

Acknowledgment. The work of the first author was supported in part by the Serbian Academy of Sciences and Arts ( $\Phi-96)$ and by the Serbian Ministry of Education, Science and Technological Development (No. \#OI 174015).

\section{References}

[1] M. Abramowitz and I.A. Stegun (Eds.) Handbook of Mathematical Functions with Formulas, Graphs, and Mathematical Tables, Dover, New York, 1972.

[2] W.N. Bailey, Products of generalized hypergeometric series, Proc. Lond. Math. Soc. s2-28 (1), 242-254, 1928.

[3] J. Choi and A.K. Rathie, Quadratic transformations involving hypergeometric functions of two and higher order, East Asian Math. J. 22, 71-77, 2006.

[4] W. Chu, Telescopic approach to a formula of ${ }_{2} F_{1}$-series by Gosper and Ebisu, Proc. Japan Acad. Ser. A Math. Sci. 93 (3), 13-15, 2017.

[5] A. Ebisu, On a strange evaluation of the hypergeometric series by Gosper, Ramanujan J. 32 (1), 101-108, 2013.

[6] H. Exton, Quadratic transformations involving hypergeometric functions of higher order, Ganita 54, 13-15, 2003.

[7] I. Gessel and D. Stanton, Strange evaluations of hypergeometric series, SIAM J. Math. Anal. 13 (2), 295-308, 1982.

[8] R.Wm. Gosper, Private Communication to Richard Askey, Dec. 21, 1977.

[9] Y.S. Kim, A.K. Rathie and R.B. Paris, A note on a hypergeometric transformation formula due to Slater with application, Math. Aeterna, 5 (1), 217-223, 2015.

[10] G.V. Milovanović, R.K. Parmar and A.K. Rathie, A study of generalized summation theorems for the series ${ }_{2} F_{1}$ with an applications to Laplace transforms of convolution type integrals involving Kummer's functions ${ }_{1} F_{1}$, Appl. Anal. Discrete Math. 12 (1), 257-272, 2018.

[11] T. Pogany and A.K. Rathie, Extension of a quadratic transformation due to Exton, Appl. Math. Comput. 215, 423-426, 2009.

[12] M.A. Rakha and A.K. Rathie, Generalizations of classical summation theorems for the series ${ }_{2} F_{1}$ and ${ }_{3} F_{2}$ with applications, Integral Transforms Spec. Funct. 229 (11), 823-840, 2011. 
[13] L.J. Slater, Generalized Hypergeometric Functions, Cambridge Univ. Press, Cambridge, 1966.

[14] H.M. Srivastava and H.L. Manocha, A Treatise on Generating Functions, Ellis Horwood Ltd., Chichester, 1984. 